\title{
BMJ Open International mixed methods study protocol to develop a patient-reported outcome measure for all types of chronic wounds (the WOUND-Q)
}

\author{
Anne Klassen (D) , ${ }^{1}$ Emiel LWG van Haren, ${ }^{2}$ Karen Cross, ${ }^{3}$ Kenneth L Fan, ${ }^{4}$ \\ Chris Gibbons, ${ }^{5}$ Maarten M Hoogbergen,, ${ }^{2}$ Natasha M Longmire, ${ }^{1}$ Lotte Poulsen, ${ }^{6}$ \\ Jens Ahm Sorensen, ${ }^{7}$ Lee Squitieri, ${ }^{8}$ Elena Tsangaris, ${ }^{5}$ Tert $\mathrm{C}$ van Alphen, ${ }^{2}$ \\ Anne-Margreet van Dishoeck, ${ }^{9}$ Dali Vasilic, ${ }^{9}$ Andrea L Pusic ${ }^{5}$
}

To cite: Klassen $\mathrm{A}$, van Haren ELWG, Cross K, et al. International mixed methods study protocol to develop a patient-reported outcome measure for all types of chronic wounds (the WOUND-Q). BMJ Open 2020;10:e032332. doi:10.1136/ bmjopen-2019-032332

- Prepublication history for this paper is available online. To view these files, please visit the journal online (http://dx.doi. org/10.1136/bmjopen-2019032332).

Received 03 July 2019

Revised 03 January 2020

Accepted 15 January 2020

Check for updates

(C) Author(s) (or their employer(s)) 2020. Re-use permitted under CC BY-NC. No commercial re-use. See rights and permissions. Published by BMJ.

For numbered affiliations see end of article.

Correspondence to

Dr Andrea L Pusic;

apusic@bwh.harvard.edu

\section{ABSTRACT}

Introduction Most patient-reported outcome measures (PROM) for chronic wounds are specific to a single wound type (eg, pressure ulcer) or part of the body. A barrier to outcome assessment in wound care and research is the lack of a rigorously designed PROM that can be used across wound types and locations. This mixed method study describes the protocol for an international collaboration to develop and validate a new PROM called the WOUND-Q for adults with chronic wounds.

Methods and analysis In phase I, the qualitative approach of interpretive description is used to elicit concepts important to people with wounds regarding outcome. Participants from Canada, Denmark, the Netherlands, and the USA are aged 18 years and older and have a wound that has lasted 3 months or longer. Interviews are digitally recorded, transcribed and coded. A conceptual framework and preliminary item pool are developed from the qualitative dataset. Draft scales are formed to cover important themes in the conceptual framework. These scales are refined using feedback from people with chronic wounds and wound care experts. After refinement, the scales are translated into Danish and Dutch, following rigorous methods, to prepare for an international field-test study. In phase II, data are collected in Canada, Denmark, the Netherlands, and the USA. An international sample of people with a large variety of chronic wounds complete the WOUND-Q. Rasch Measurement Theory analysis is used to identify the best subset of items to retain for each scale and to examine reliability and validity.

Ethics and dissemination This study is coordinated at Brigham and Women's Hospital (Boston, USA). Ethics board approval was received at each participating site for both study phases. Findings will be published in peer-reviewed journals and presented at national and international conferences and meetings.

\section{INTRODUCTION}

Each year, millions of individuals require treatment for chronic wounds. A recent systematic review of 11 studies reported a prevalence in

\section{Strengths and limitations of this study}

- Recruitment of an international sample makes it possible to develop a patient-reported outcome measure (PROM) that reflects the concerns of patients in multiple countries

- Including people with varying types of chronic wounds in different locations on the body ensures that the WOUND-Q is broadly applicable.

- We adhere to published guidelines for PROM development, including rigorous methods for translation and cultural adaptation.

- We use a modern psychometric approach (Rasch Measurement Theory) to enhance the interpretability of WOUND-Q scores.

- A limitation of our study is that patient involvement does not include membership in the research team. Another limitation is that the WOUND-Q field-test takes place only in high-income countries.

the general population to be 2.21 and 1.51 per 1000 population for wounds of mixed aetiology and chronic leg ulcers respectively. ${ }^{1}$ Wound care has a huge economic impact on healthcare systems worldwide. An analysis of US Medicare claims for 2014 showed that $15 \%$ of beneficiaries ( 8.2 million) had an episode of care for a chronic wound or infection, with costs estimated between 28.1 and 96.8 billion. $^{2}$ In the UK, a study estimated that $4 \%$ of the total expenditure by the National Health Service in 2012/13 ( 5.3 billion pounds) went towards managing chronic wounds and associated morbidity for 2.2 million patients. ${ }^{3}$

Chronic wounds have many different causes and numerous treatment modalities. The Cochrane Wounds review group, established in 1995, lists more than 150 protocols and reviews of the effects of interventions to prevent and treat wounds and their 
complications. ${ }^{4}$ Outcome measures used in intervention studies tend to involve the use of objective measures (eg, healed wounds, rate of healing, and adverse effects). The inclusion of carefully designed patient-reported outcome measures (PROMs) that ask about concerns that matter the most to patients, including bothersome symptoms, such pain, exudate and odour, and the impact of wounds on aspects of quality of life, can provide important additional information from the patient perspective. Cochrane reviews of wound treatments show that such outcomes are often overlooked in treatment studies. ${ }^{5-8}$.

PROMs that measure outcomes that matter to patient, as well as their experience of healthcare, are increasingly used to inform quality improvement initiatives, patient care, and comparative effectiveness research. ${ }^{9-11}$ In chronic wounds, four reviews of PROMs have been published. ${ }^{12-15}$ These reviews report that generic tools (eg, SF-36, EQ-5D, and Nottingham Health Profile) are often used. Such tools are limited in terms of content validity as they fail to ask about important wound-specific issues (eg, odour and exudate). In terms of woundspecific PROMs, most were developed for a specific type of wound, including venous leg ulcers, ${ }^{16-22}$ foot ulcers, ${ }^{23} 24$ and pressure ulcers, ${ }^{25} 26$ or for wounds on specific parts of the body. ${ }^{27}$ The Wound-QoL ${ }^{28}$ (Questionnaire on quality of life with chronic wounds) represents an exception, as it was designed for all types of chronic wounds. This 17-item instrument, published in 2014, was developed by taking items from three existing PROMs, using factor analysis to determine how the items group together, and then attaching labels (body, everyday life, and psyche) to each concept. The Wound-QoL can be scored separately for each scale or by adding together the scales for a total score. PROMs that add scales together for a total score can be problematic because the concept of interest measured by the instrument may not be clear. The same is true for PROMs that score each scale separately if the scales are composed of item sets that ask about multiple concepts of interest (COI). For example, the body scale from the Wound-QoL has five items that measures a range of concepts, including pain, wound discharge and problems sleeping. ${ }^{28}$ Item sets that ask about multiple concepts are limited in their ability to measure change in specific issues. In the context of a clinical trial, such scales can mask effects of treatment (eg, when the direction and size of the scores vary by concept). The alternative approach is to design a set of independently functioning scales that each measure a unidimensional construct, for example, discharge, smell, sleep interference, and so on. An advantage of this approach is that respondent burden can be decreased as only the scales that are relevant to a particular research question or clinical scenario need to be used.

Currently, there is no comprehensive PROM designed using a modern psychometric approach covering all type of chronic wounds that are located anywhere on the body. The modern psychometric approach uses more sophisticated models and techniques than the traditional approach, providing more diagnostic details that aid in the refinement of scales that have interval (rather than ordinal) measurement properties. ${ }^{29} 30$ This protocol describes an international collaboration between investigators in Canada, Denmark, the Netherlands, and the USA that aims to develop a new PROM (ie, the WOUND-Q) for patients with chronic wounds. The WOUND-Q will contain a comprehensive set of independently functioning scales designed to measure outcomes that matter to patients with any type of chronic wound, as well as scales to measure patients experience of wound care. We describe the mixed methods approach that we previously published in the development of other Q-Portfolio instruments. ${ }^{31-33}$

\section{METHODS AND ANALYSIS}

Development of the WOUND-Q follows guidelines for PROM development outlined by the Scientific Advisory Committee of the Medical Outcomes Trust, ${ }^{34}$ the USA Food and Drug Administration, ${ }^{35}$ and the International Society for Pharmacoeconomics and Outcomes Research (ISPOR). ${ }^{36-38}$ We aim to develop a self-report instrument for adults ( $\geq 18$ years old) with any type of chronic wound located anywhere on the body. Our goal is to develop a comprehensive set of independently functioning scales that measure COI important to patients and healthcare providers working in chronic wound care.

Our protocol covers a multi-phase mixed methods study that includes qualitative and quantitative lines of inquiry. Figure 1 shows the three main phases involved in the development of a PROM. ${ }^{33}$ These phases include iterative steps for item generation, item reduction, and psychometric validation. Careful adherence to the steps outlined in figure 1 will ensure the WOUND-Q fulfils minimum standards for acceptable psychometric properties described by the International Society for Quality of Life Research ${ }^{39}$ and the Consensus-based Standards for the Selection of Health Status Measurement Instruments. ${ }^{4041}$

\section{Phase I: qualitative}

We take a qualitative approach called interpretive description. ${ }^{42}{ }^{43}$ In our context, this applied health services approach builds on existing wound-specific theoretical knowledge, clinical knowledge, and scientific research.

\section{Sample}

Participants are purposively sampled to include a heterogeneous sample that varies by the following characteristics: age (18 years and older), gender, wound type, wound location, phase in the healing process, and risk of poor outcome (smokers and people with comorbid conditions such as diabetes and obesity). Participants are recruited in wound care clinics by a member of the healthcare team who obtain informed consent and pass contact details to a member of the research team to schedule interviews. Phase 1 involves sites from Canada (University of St 


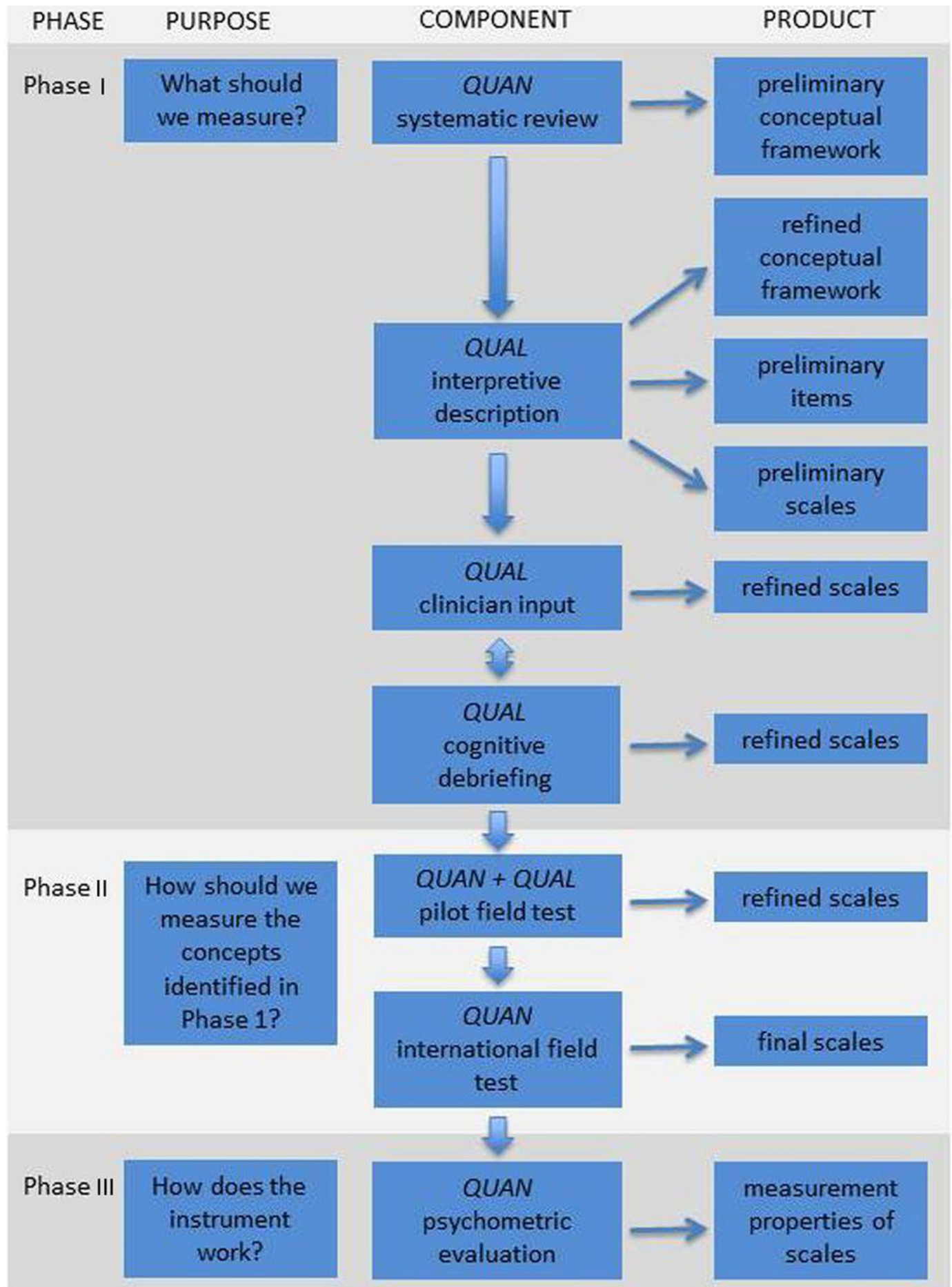

Figure 1 Flow diagram illustrating the multiphase mixed methods approach to the development of the WOUND-Q. QUAN, quantitative study component; QUAL, qualitative study component. Image reproduced from Wong Riff et al. ${ }^{33}$

Michael's College, Toronto), Denmark (Odense University Hospital, Odense), the Netherlands (Catharina Hospital, Eindhoven; DaVinci Wound Clinic, Geldrop) and the USA (University of California, Los Angeles Berkley East Nursing Home, Berkley, and University of California, Los Angeles Medical Center, Santa Monica). Interviews are conducted face-to-face or by phone depending on participant preference and logistics for travel.

\section{Concept elicitation}

An interview guide (see box 1) is used to guide the interviews. Topics are informed by published wound-specific PROMs in the literature. ${ }^{13-15} 2528$ Interviews are audiorecorded and transcribed verbatim. Interviews performed in Denmark and the Netherlands are translated into English by professional translators and are coded by the local research team members. Data are coded line-by-line 


\section{Box 1 WOUND-Q interview guide}

\section{Wound}

1. How did your wound originate? Probe: any comorbid diabetes and obesity.

2. Can you tell me about any wounds you've had in the past?

3. How was the healing process for you? Probe: haemostasis, inflammatory, proliferation, and remodelling/maturation.

\section{Treatments}

4. What treatment(s) have you had for your wound?

5. What was good/bad about the treatment(s)? Probe: side effects.

\section{Symptoms}

6. Can you describe any symptoms you experience? Probe: for example, pain, odour and exudate.

7. Have symptoms changed over time? Probe: haemostasis, inflammation, proliferation and remodelling.

8. How bothersome are the symptoms?

9. How do you cope with the symptoms?

\section{Recovery}

10. How quickly did you recover?

11. What was the recovery process like? Probe: haemostasis, inflammation, proliferation and remodelling.

12. Can you describe the early life impact? Probe: impact on physical, social, emotional and social life.

\section{Appearance}

13. How would you describe the appearance of your wound? Probe: for descriptive detail.

14. What do you dislike about the appearance of your wound?

15. How has your appearance changed? Probe: haemostasis, inflammation, proliferation and remodelling.

16. Is there anything about the wound you wish looked different? Probe: for descriptive detail.

17. Do/did you ever hide or cover your wound? How do you do this?

\section{Physical function}

18. Does the wound create physical issues? Probe: for example, mobility and activity limitations.

19. How have these physical issues changed? Probe: haemostasis, inflammation, proliferation and remodelling.

\section{Psychological well-being}

20. How does your wound affect how you feel? Probe: happy, sad, anxious, frustrated and self-conscious.

21. How does your wound affect how you feel about yourself? Probe: self-esteem, body image and confidence.

22. How have your emotions changed? Probe: haemostasis, inflammation, proliferation and remodelling.

\section{Social Life}

23. What has it been like for you at home? Probe: partner, family and children.

24. Has the wound affected your usual activities? Probe: work/ education.

25. Do people ever comment on your wound? Probe: how did you react and how did you feel?

26. Are there things you would have liked to do but do not because of your wound?

27. Has anyone ever treated you differently because of your wound? Probe: friends, family and strangers.

\section{Box 1 Continued}

28. How else does your wound affect your social life? Probe: with friends, meeting new people and dating.

\section{Experience of care}

29. Who did you see at the hospital or clinic? Probe: doctor, nurse, receptionist and so on.

30. What are the people like who cared for you? Probe: friendly, made you feel comfortable, easy to talk to, listened to you, respectful and available.

31. What kind of verbal and written information did you receive? Probe: gave enough information, let you ask questions, answered your questions, information about recovery and treatment information.

32. What things could the healthcare team do differently to improve the care you received?

33. What should the perfect wound healing centre be like?

\section{Other questions}

34. Is there anything I have not asked you that you think it is important for me to know?

whereby participant quotes are labelled with top-level domains, themes, and subthemes. Data are moved from Word to Excel for analysis. Participant characteristics are included in Excel to identify common and unique COI by participant characteristics (eg, wound type and location). Data analysis is done concurrently with data collection to add new concepts to the interview guide for probing with new participants. Sampling and recruitment continue until the point of saturation is reached, that is, no further new concepts are elicited from additional interviews. ${ }^{44}$

Rigour in the qualitative phase of the study is ensured by having one team member code the qualitative transcripts and a second team member confirm the codes. Also, performing qualitative interviews and analysis at the same time makes it possible to add COIs important to participants to the interview guide in order to explore if the COIs are important to participants in subsequent interviews. Finally, peer debriefing is performed to verify the analysis of the qualitative data between team members who perform the coding, as well as with the full research team at the research team meeting described below.

Qualitative analysis leads to the refinement of a conceptual framework covering the main COI of people with chronic wounds. This framework is used to guide scale development.

\section{Item generation}

Participant quotes are used to create a comprehensive item pool. Items retain the language of participants as much as possible to ensure that scale content is easy to understand and resonates with patient experience. The item pool is sorted and analysed by levels of coding (ie, top-level domains and theme/subthemes) and participant characteristics (eg, wound type) to identify common and unique COI. The item pool is used to develop a comprehensive set of independently functioning scales that cover key aspects of the conceptual framework. 


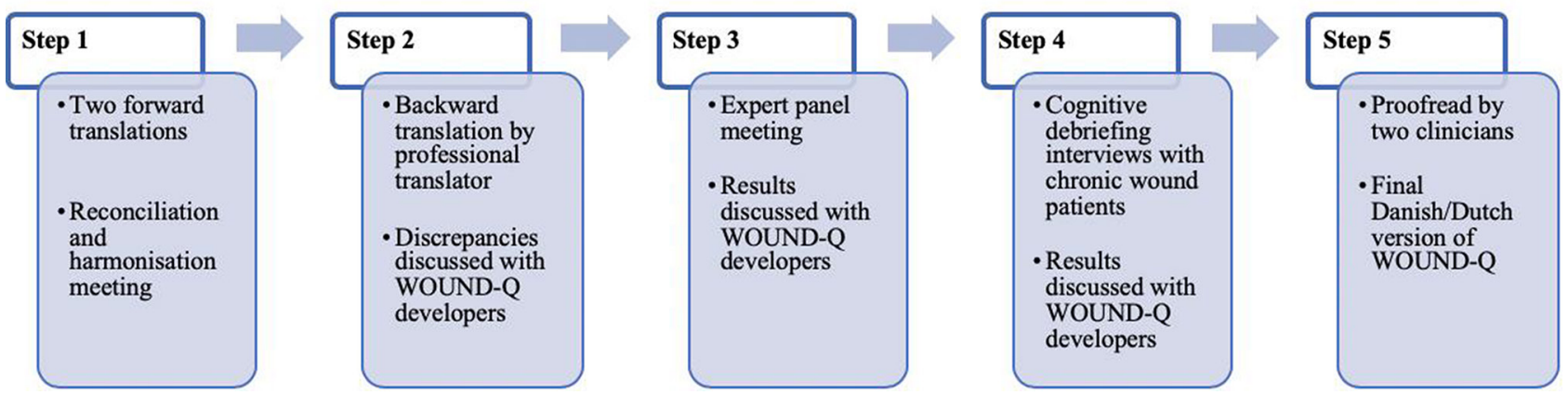

Figure 2 Translation and cultural adaption steps for the WOUND-Q. Image reproduced from van Alphen et al..$^{51}$ QUAN, quantitative study component; QUAL, qualitative study component.

\section{Scale development}

Scale development is informed by the Rasch Measurement Theory (RMT) approach. ${ }^{30} 45$ In this approach, a pool of items that are reflective of the underlying constructs are derived from the qualitative data to create, for each scale, a conformable set of items that together map out a construct on a clinical hierarchy. Later in the study (phase II), the field-test data are analysed to see if the theorised construct is supported by the data, that is, do the data 'fit' the Rasch model. The pattern expected by the Rasch model follows a strict deterministic hierarchical ordering of items called Guttman scaling. When the data fit the Rasch model, the estimates derived from the model are considered appropriate, and it is legitimate to sum the items in a scale to obtain a total score that provides interval-level measurement. Scales are assigned appropriate instructions and a time frame for reporting. Each scale is assigned four or five labelled response options to keep them simple and in line with published guidelines. ${ }^{46}$

\section{Research team meeting}

After half the interviews are conducted and fully analysed, a full day face-to-face research team meeting is held to review the sample characteristics and data findings in order to identify and address gaps and issues. At the meeting, wound care experts and the research team reviews codes, the item pool, and drafts scales that cover key aspects of the preliminary conceptual framework. Following this meeting, interviews and analysis continue until no new concepts are elicited from subsequent interviews.

\section{Scale refinement}

Scales are refined through multiple rounds of cognitive interviews ${ }^{478}$ using the 'think aloud' method. ${ }^{49}$ The aim is to determine content validity, that is whether scale content is relevant, comprehensive, and comprehendible. ${ }^{41}$ Participants from the initial interviews are invited to review the scales (English versions). Interviews are audio-recorded, transcribed, and analysed line-by-line. Feedback on instructions, response options, and items are examined and used to revise the scales. Participants are encouraged to suggest missing issues that can be developed into items and added to scales.

Between rounds of cognitive interviews, the WOUND-Q is shown to experts for feedback. A web-based secure Research Electronic Data Capture (REDCap) survey is designed. ${ }^{50}$ An international sample of wound experts are emailed the link to access the survey and provide feedback on the instructions, response options, and items, and to suggest missing content that could be formed into new items. One reminder email is sent after 10 days. Feedback provided by experts is used to revise the scales.

\section{Translations}

The WOUND-Q is translated into Danish and Dutch following steps outlined in figure $2 .{ }^{51}$ Translations follow guidelines set forth by the ISPOR ${ }^{38}$ and the World Health Organization. ${ }^{52}$ These guidelines outline a rigorous process, previously used by our team, ${ }^{53}$ which involves two independent forward and one backward translation, an expert panel meeting, and a series of cognitive debriefing interviews with patients with chronic wounds. The aim is to create conceptually equivalent translations rather than literal translations. Producing more than one translation at the same time makes it possible to revise the items, instructions, and response options of the WOUND-Q based on feedback from the translation work and to harmonise the translations by comparing the Danish and Dutch with each other and with the English version.

\section{Phase II: quantitative}

The phase II field-test study begins with a pilot field-test sample to identify any final changes to the scales that are needed. Data from the first 250 participants are used to examine the psychometric performance of each scale.

Phase II involves collection of data from a large international sample of patients with wounds from sites in Canada (University of St Michael's, Toronto), Denmark (Odense University Hospital, Odense), the Netherlands (Catharina Hospital, Eindhoven; DaVinci Wound Clinic, Geldrop; Erasmus Medical Center, Rotterdam) and the USA (Brigham and Women's Hospital, Boston; Massachusetts General Hospital Boston; University of California, Los Angeles Berkley East Nursing Home, Berkley; University of California, Los Angeles Medical Center, 
Santa Monica; MedStar Georgetown University Hospital, and MedStar Washington Hospital Center, Washington). Additional sites can be added if needed to ensure that the sample is large enough to explore how the items and scales function at the subgroup level.

Participants are aged 18 years and older, cognitively able to self-report, and have one or more chronic wounds anywhere on their body. A chronic wound is defined as a wound that has lasted 3 months or longer. Patients are recruited in hospital clinics by research assistants who obtain informed consent. Data are collected using tablets with data entered into databases. REDCap databases are hosted at Brigham and Women's Hospital in Boston (for the Canadian and US data), and at Odense University Hospital (for Danish data). In the Netherlands, data are collected using the Castor database. Instructions with branching logic are used to ensure that only correct scales are administered to reduce respondent burden.

Data collected via separate databases are merged using IBM SPSS Statistics V.26 and are formatted appropriately for RUMM2030 software for RMT analysis. ${ }^{54}$ The RMT analysis is used to refine each scale and to create the scoring algorithms that future users of the scales will require. A range of evidence is used to evaluate each item in a scale and examine how the items function as a set. Items that are the most effective in measuring the concepts measured by a scale are retained. Items that do not perform well are removed. Decisions based on the following set of statistical and graphical tests are used, and described in detail elsewhere ${ }^{30}$ :

Thresholds for item response options: We examine thresholds between response options (eg, 'not at all' and 'a little') to determine if a scales response categories are ordered, meaning that a ' 1 ' on a 4-point scale sits lower down a continuum than a ' 2 ' and so on. Items with disordered thresholds may be dropped or recoded to ensure thresholds are properly ordered.

Item fit statistics: Three fit indicators are inspected including $\log$ residuals (item-person interaction), $\chi^{2}$ values (item-trait interaction) and item characteristic curves. As a guide, ideal fit residuals are between -2.5 and +2.5 , with Bonferroni adjusted $\chi^{2}$ values non-significant. The three fit indicators are interpreted together to decide which items to retain or drop.

Dependency: Residual correlations between pairs of items are inspected to identify high residual correlations as these can inflate reliability. For high residual correlations, a subtest is performed to determine the impact on the person separation index reliability statistic.

Targeting: The location of items is examined to determine if they are evenly spread over a reasonable range that matches the range of the construct reported by the sample. There should be minimal evidence of a floor or ceiling effect so that people with a wide variety of wounds at different stages of healing can be effectively measured over time.

Differential item functioning (DIF): DIF is examined using analysis of variance of item residuals to determine if individuals in subgroups (eg, sex, age, country and type of wound) respond differently to items despite the same measured trait level. We choose random samples to create equal-sized subgroups. Items with $\chi^{2}$ values significant after Bonferroni adjustment are split on the variable that evidences DIF, and the new and original person locations are correlated to examine impact on scoring.

Unidimensionality: We determine if all items in a scale measure the same, single latent construct using the method proposed by Smith based on independent t-tests. $^{55}$

Person separation index: This reliability statistic measures error associated with the measurement of people in a sample and is similar in interpretation to Cronbach $\alpha .{ }^{56}$

The minimum number of people needed to perform RMT analysis is 150 to have 50 respondents in each of 3 class intervals for the $\chi^{2}$ analysis for tests of item fit. ${ }^{57}$ To examine DIF by country will require 600 participants ( $\mathrm{n}=150$ per country). Our target is 250 per country to provide an even more robust scoring algorithms and normative scores.

In IBM SPSS Statistics V.26, traditional psychometric properties are examined including reliability (internal consistency), and construct validity. Once item reduction and psychometric validation of the field-test data is completed, the WOUND-Q will be made available for licensing through the Q-Portfolio website (www.qportfolio.org).

\section{Subsequent phases}

The phase II field-test study is currently ongoing and will be completed in 2020. We plan to seek grant funding to conduct a phase III study to examine further measurement properties on the item-reduced scales, such as concurrent validity (the degree to which scores on an instrument correlate with the Wound-QoL ${ }^{27}$ ), test-retest reliability, and to determine each scales' ability to measure clinical change following wound treatment using anchor-based and distribution-based methods. ${ }^{58-60}$ These studies will be planned to reflect priorities identified by wound care teams and will use our international network of wound care centres.

\section{Patient and public involvement}

A limitation of our study is that patient involvement does not include membership in the research team. However, our approach to PROM development is patient oriented as we engage a large number of people with wounds, as well as clinical experts, in all stages of our research. Input from patients with chronic wounds are indispensable to ensuring that the scales are developed to measure outcomes that matter to them in the language they use so that the final instrument resonates. Patients who take part in qualitative interviews are invited to participate in the cognitive interviews as continuity of involvement ensures the scales accurately reflect the experiences of patients living with chronic wounds. We recognise a limitation of using the same participants twice could be that others not involved in the 
initial phase may provide new insights. Experts are involved at different steps in the research, including a research meeting half way through the qualitative interview phases, as well as during scale refinement phase. Involvement of experts ensures that the scales will cover all clinically relevant outcomes and experiences of care.

Participants provide both written and oral consent before participating in an interview, and written consent for participating in the field-test study. Participants in phase I are asked to discuss issues that can be sensitive, and they may experience distress. If necessary, participants will be put in touch with a healthcare provider at the recruiting site to obtain support. Participant data is de-identified during transcription for the qualitative interviews. All data collected are kept secure and confidential following institution rules governing research data storage.

\section{DISSEMINATION}

The WOUND-Q will be made available free of charge to all non-profit users. Our team will promote uptake of the WOUND-Q among stakeholder groups including researchers, healthcare practitioners, decision makers and policy makers. Our dissemination initiatives will include face-to-face interactions such as presentations at national and international meetings, as well as electronic and hardcopy media, including publication in journals that are valued and read by our target audiences. The Q-Portfolio website (www.qportfolio.org) and social media (eg, Twitter and Instagram) will be used to spread awareness of the WOUND-Q to our network of followers.

\section{Author affiliations}

${ }^{1}$ Pediatrics, McMaster University, Hamilton, Ontario, Canada

${ }^{2}$ Plastic and Reconstructive Surgery, Catharina Ziekenhuis, Eindhoven, The Netherlands

${ }^{3}$ Plastic Surgery, St Michael's Hospital, Toronto, Ontario, Canada

${ }^{4}$ Plastic and Reconstructive Surgery, MedStar Georgetown University Hospital, Washington, DC, USA

${ }^{5}$ Department of Surgery, Patient Reported Outcome, Value, and Experience (PROVE) Center, Harvard Medical School, Brigham and Women's Hospital, Boston, Massachusetts, USA

${ }^{6}$ Department of Plastic Surgery, University of Southern Denmark, Odense, Denmark ${ }^{7}$ Department of Plastic Surgery, Odense University Hospital, Odense, Denmark ${ }^{8}$ Division of Plastic and Reconstructive Surgery, University of Southern California, Los Angeles, California, USA

${ }^{9}$ Plastic and Reconstructive Surgery, Erasmus Medical Center, Rotterdam, The Netherlands

\section{Twitter Anne Klassen @anneklassen}

Contributors AK and ALP conceived and designed the study. The other authors are involved in the set up and acquisition of data from their sites. AK, ELWGVH, KC, KLF, CG, MMH, NML, LP, JAS, LS, ET, TCVA, A-MvD, DV and ALP revised the article critically for important intellectual content and have approved the final version to be published.

Funding Phases I and II of this study are funded by research grants received from the Plastic Surgery Foundation (120593). The authors have no financial interest to declare in relation to the content of this article. The Article Processing Charge was paid from the Plastic Surgery Foundation grant.

Competing interests The WOUND- $Q$ will be owned by Memorial Sloan-Kettering Cancer Center. Drs ALP and AK are codevelopers of other Q-Portfolio instruments and receive a share of any license revenue on the inventor sharing policies of the institutions that own them.

Patient consent for publication Not required.

Ethics approval This study is coordinated at Brigham and Women's Hospital (Boston, USA). Ethics board approval was obtained from sites in Canada (University of St. Michael's, Toronto), the Netherlands (Catharina Hospital, Eindhoven; DaVinci Wound Clinic, Geldrop; Erasmus Medical Center, Rotterdam) and the USA (Brigham and Women's Hospital, University of California, Los Angeles Berkley East Nursing Home, and University of California, Los Angeles Medical Center, MedStar Georgetown University Hospital, and MedStar Washington Hospital Center). For Odense University Hospital in Denmark, we obtained permission at the data protecting agency since ethics is not required for studies that involve questionnaire surveys in Denmark.

Provenance and peer review Not commissioned; externally peer reviewed.

Open access This is an open access article distributed in accordance with the Creative Commons Attribution Non Commercial (CC BY-NC 4.0) license, which permits others to distribute, remix, adapt, build upon this work non-commercially, and license their derivative works on different terms, provided the original work is properly cited, appropriate credit is given, any changes made indicated, and the use is non-commercial. See: http://creativecommons.org/licenses/by-nc/4.0/.

ORCID iD

Anne Klassen http://orcid.org/0000-0003-4720-0096

\section{REFERENCES}

1 Martinengo L, Olsson M, Bajpai R, et al. Prevalence of chronic wounds in the general population: systematic review and metaanalysis of observational studies. Ann Epidemiol 2019;29:8-15.

2 Nussbaum SR, Carter MJ, Fife CE, et al. An economic evaluation of the impact, cost, and Medicare policy implications of chronic nonhealing wounds. Value Health 2018;21:27-32.

3 Guest JF, Ayoub N, Mcllwraith T, et al. Health economic burden that wounds impose on the National health service in the UK. BMJ Open 2015;5:e009283.

4 Cochrane Wounds. Cochrane Ireland and Cochrane UK symposium 2020. Available: https://wounds.cochrane.org/ [Accessed May 2019].

5 Smith F, Dryburgh N, Donaldson J, et al. Debridement for surgical wounds. Cochrane Database Syst Rev 2013;9:CD006214.

6 Kranke P, Bennett M, Roeckl-Wiedmann I, et al. Hyperbaric oxygen therapy for chronic wounds. Cochrane Database Syst Rev 2004;2:CD004123.

7 Norman G, Westby MJ, Rithalia AD, et al. Dressings and topical agents for treating venous leg ulcers. Cochrane Database Syst Rev 2018;6:CD012583.

8 Dumville JC, Webster J, Evans D, et al. Negative pressure wound therapy for treating pressure ulcers. Cochrane Database Syst Rev 2015;5:CD011334.

9 Calvert M, Kyte D, Price G, et al. Maximising the impact of patient reported outcome assessment for patients and society. BMJ 2019;364:k5267.

10 Nelson EC, Eftimovska E, Lind C, et al. Patient reported outcome measures in practice. BMJ 2015;350:g7818.

11 Black N. Patient reported outcome measures could help transform healthcare. BMJ 2013;346:f167.

12 Poku E, Aber A, Phillips P, et al. Systematic review assessing the measurement properties of patient-reported outcomes for venous leg ulcers. BJS Open 2017;1:138-47.

13 Gorecki C, Nixon J, Lamping DL, et al. Patient-Reported outcome measures for chronic wounds with particular reference to pressure ulcer research: a systematic review. Int J Nurs Stud 2014;51:157-65.

14 González-Consuegra RV, Verdú J. Quality of life in people with venous leg ulcers: an integrative review. J Adv Nurs 2011;67:926-44.

15 Palfreyman SJ, Tod AM, Brazier JE, et al. A systematic review of health-related quality of life instruments used for people with venous ulcers: an assessment of their suitability and psychometric properties. J Clin Nurs 2010;19:2673-703.

16 Hyland ME, Ley A, Thomson B. Quality of life of leg ulcer patients: questionnaire and preliminary findings. J Wound Care 1994;3:294-8.

17 Hareendran A, Doll H, Wild DJ, et al. The venous leg ulcer quality of life (VLU-QoL) questionnaire: development and psychometric validation. Wound Repair Regen 2007;15:465-73.

18 Smith JJ, Guest MG, Greenhalgh RM, et al. Measuring the quality of life in patients with venous ulcers. J Vasc Surg 2000;31:642-9. 
19 Venkatraman PD, Anand SC, Dean C, et al. Pilot study investigating the feasibility of an ulcer-specific quality of life questionnaire. Phlebology 2005;20:14-27.

20 Palfreyman S, Michaels J, Brazier J. Development of a tool to examine the effect of venous ulcers on patients' quality of life. Nurs Stand 2007;21:57-69.

21 Bland JM, Dumville JC, Ashby RL, et al. Validation of the VEINESQOL quality of life instrument in venous leg ulcers: repeatability and validity study embedded in a randomised clinical trial. BMC Cardiovasc Disord 2015;15:85.

22 Brown A, Kendall S, Flanagan M, et al. Encouraging patients to selfcare - the preliminary development and validation of the VeLUSETC, a self-efficacy tool for venous leg ulcer patients, aged 60 years and over. Int Wound J 2014;11:326-34.

23 Abetz L, Sutton M, Brady L, et al. The diabetic foot ulcer scale (DFS): a quality of life instrument for use in clinical trials. Pract Diab Int 2002;19:167-75.

24 Vileikyte L, Peyrot M, Bundy C, et al. The development and validation of a neuropathy- and foot ulcer-specific quality of life instrument. Diabetes Care 2003;26:2549-55.

25 Gorecki C, Brown JM, Cano S, et al. Development and validation of a new patient-reported outcome measure for patients with pressure ulcers: the PU-QOL instrument. Health Qual Life Outcomes 2013;11:95.

26 Kisala PA, Tulsky DS, Choi SW, et al. Development and psychometric characteristics of the SCl-QOL pressure ulcers scale and short form. J Spinal Cord Med 2015;38:303-14.

27 Price P, Harding K. Cardiff wound impact schedule: the development of a condition-specific questionnaire to assess health-related quality of life in patients with chronic wounds of the lower limb. Int Wound $J$ 2004:1:10-17.

28 Blome C, Baade K, Debus ES, et al. The "Wound-QoL": a short questionnaire measuring quality of life in patients with chronic wounds based on three established disease-specific instruments. Wound Repair Regen 2014;22:504-14.

29 Petrillo J, Cano SJ, McLeod LD, et al. Using classical test theory, item response theory, and Rasch measurement theory to evaluate patient-reported outcome measures: a comparison of worked examples. Value Health 2015;18:25-34.

30 Hobart J, Cano S. Improving the evaluation of therapeutic interventions in multiple sclerosis: the role of new psychometric methods. Health Technol Assess 2009;13:1-177.

31 Sierakowski K, Dean NR, Pusic AL, et al. International multiphase mixed methods study protocol to develop a cross-cultural patientreported outcome and experience measure for hand conditions (HAND-Q). BMJ Open 2019;9:e025822.

32 Klassen AF, Kaur M, Johnson N, et al. International phase I study protocol to develop a patient-reported outcome measure for adolescents and adults receiving gender-affirming treatments (the GENDER-Q). BMJ Open 2018;8:e025435.

33 Wong Riff KWY, Tsangaris E, Goodacre T, et al. International multiphase mixed methods study protocol to develop a crosscultural patient-reported outcome instrument for children and young adults with cleft lip and/or palate (CLEFT-Q). BMJ Open 2017;7:e015467.

34 Aaronson N, Alonso J, Burnam A, et al. Assessing health status and quality-of-life instruments: attributes and review criteria. Qual Life Res 2002;11:193-205.

35 US Department of Health and Human Services. Guidance for industry patient-reported outcome measures: use in medical product development to support labeling claims. Available: https://www.fda. gov/downloads/drugs/guidances/ucm193282.pdf [Accessed May 2019].

36 Patrick DL, Burke LB, Gwaltney CJ, et al. Content validity-establishing and reporting the evidence in newly developed patient-reported outcomes (PRO) instruments for medical product evaluation: ISPOR PRO good research practices task force report: part 1--eliciting concepts for a new PRO instrument. Value Health 2011:14:967-77.

37 Patrick DL, Burke LB, Gwaltney CJ, et al. Content validity-establishing and reporting the evidence in newly developed patient-reported outcomes (PRO) instruments for medical product evaluation: ISPOR PRO Good Research Practices Task Force report: part 2--assessing respondent understanding. Value Health 2011;14:978-88.

38 Wild D, Grove A, Martin M, et al. Principles of good practice for the translation and cultural adaptation process for patient-reported outcomes (pro) measures: report of the ISPOR Task force for translation and cultural adaptation. Value Health 2005;8:94-104.

39 Reeve BB, Wyrwich KW, Wu AW, et al. ISOQOL recommends minimum standards for patient-reported outcome measures used in patient-centered outcomes and comparative effectiveness research. Qual Life Res 2013;22:1889-905.

40 Mokkink LB, Terwee CB, Patrick DL, et al. The COSMIN checklist for assessing the methodological quality of studies on measurement properties of health status measurement instruments: an international Delphi study. Qual Life Res 2010;19:539-49.

41 Terwee CB, Prinsen CAC, Chiarotto A, et al. COSMIN methodology for evaluating the content validity of patient-reported outcome measures: a Delphi study. Qual Life Res 2018;27:1159-70.

42 Thorne S, Kirkham SR, MacDonald-Emes J. Interpretive description: a noncategorical qualitative alternative for developing nursing knowledge. Res Nurs Health 1997;20:169-77.

43 Thorne SE. Interpretive description. developing qualitative inquiry. 2. Walnut Creek (CA): Left Coast Press, 2008.

44 Given LM, Sandelowski M. Theoretical Saturation. In: Given LM, ed. The SAGE encyclopedia of qualitative research methods. Thousand Oaks: SAGE Publications, Inc, 2008: 876

45 Rasch G. Studies in mathematical psychology: 1. Probabilstic models for some intelligence and attainment tests. Copenhagen: Danmarks pædagogiske Institut, 1960.

46 Khadka J, Gothwal VK, McAlinden C, et al. The importance of rating scales in measuring patient-reported outcomes. Health Qual Life Outcomes 2012;10:80.

47 Willis GB. Cognitive interviewing: a tool for improving questionnaire design. Thousand Oaks, CA: Sage Publications, 2005

48 Collins D. Pretesting survey instruments: an overview of cognitive methods. Qual Life Res 2003;12:229-38.

49 Van Someren M, Barnard Y, Sandberg J. The think-aloud method. London: Academic Press, 1994.

50 Harris PA, Taylor R, Thielke R, et al. Research electronic data capture (REDCap)--a metadata-driven methodology and workflow process for providing translational research informatics support. J Biomed Inform 2009:42:377-81.

51 van Alphen TC, Poulsen L, van Haren ELWG, et al. Danish and Dutch linguistic validation and cultural adaptation of the WOUND-Q, a PROM for chronic wounds. Eur J Plast Surg 2019;42:495-504.

52 WHO (World Health Organization). The process of translation and adaptation of instruments. Available: http://www.who.int/ substance_abuse/research_tools/translation/en/ [Accessed May 2019].

53 Poulsen L, Rose M, Klassen A, et al. Danish translation and linguistic validation of the BODY-Q: a description of the process. Eur J Plast Surg 2017;40:29-38.

54 Lab R. RUMM. Available: http://www.rummlab.com.au/ [Accessed 29 May 2019].

55 Smith EV. Detecting and evaluating the impact of multidimensionality using item fit statistics and principal component analysis of residuals. $J$ Appl Meas 2002;3:205-31.

56 Nunnally JC, Bernstein IH. Psychometric theory. 3rd edn. New York: McGraw-Hill, 1994

57 Sample size and item calibration or person measure stability. Available: http://www.rasch.org/rmt/rmt74m.htm [Accessed May 2019].

58 Terwee CB, Dekker FW, Wiersinga WM, et al. On assessing responsiveness of health-related quality of life instruments: guidelines for instrument evaluation. Qual Life Res 2003;12:349-62.

59 Husted JA, Cook RJ, Farewell VT, et al. Methods for assessing responsiveness: a critical review and recommendations. J Clin Epidemiol 2000;53:459-68.

60 Brozek JL, Guyatt GH, Schünemann HJ. How a well-grounded minimal important difference can enhance transparency of labelling claims and improve interpretation of a patient reported outcome measure. Health Qual Life Outcomes 2006;4:69. 